\title{
QUELQUES ASPECTS DE L'ECOLOGIE DU PLANCTON D'UN LAC DE MONTAGNE EUTROPHE : LE LAC DE PETICHET (ISERE)
}

\author{
par C. GAY
}

\begin{abstract}
L'auteur étudie l'évolution saisonnière et la répartition verticale du phytoplancton et du zooplancton d'un lac de moyenne montagne et précise l'écologie des espèces principales. La présence d'organismes dominants à caractère $\beta$-mésosaprobe tels que Fragilaria crotonensis, Asterionella formosa, Melosira italica, Daphnia longispina et Cyclops vicinus, et la formation de "fleurs d'eau" à Dinobryon divergens et Oscillatoria? cortiana soulignent les résultats des analyses physico-chimiques de l'eau qui avaient mis en évidence le caractère eutrophe du lac.
\end{abstract}

\section{Several aspects of the ecology of plankton in a eutrophic mountain lake : Lake Petichet (Isère).}

The author studied the seasonal evolution and vertical distribution of phytoplankton and zooplankton in a mountain lake at mean altitude, and described the ecology of the principal species. The presence of dominant specics from the $\beta$-mesosaprobic group, such as Fragilaria crotonensis, Asterionella formosa, Melosira italica, Daphnia longispina and Cyclops vicinus, and the formation of "water blooms" of Dinobryon divergens and Oscillatoria? cortiana supports the concusions from physical and chemical analyses of the water that the lake is eutrophic.

\section{INTRODUCTION}

Le présent travail concernant le plancton du lac de Petichet fait partie d'une étude plus vaste ayant pour but essentiel de préciser le niveau de trophie de ses eaux et l'origine de sa pollution. En effet, depuis quelques années, des signes d'eutrophisation accélérée ont été décelés. Or, les eaux du lac de Petitchet se déversent dans le Grand Lac de Laffrey qui risque, de ce fait, de suivre la même évolution. Aucune étude n'ayant été jusqu'alors consacrée à ce lac, il était nécessaire de connaître le fonctionnement de cet écosystème avant de définir les moyens de protection à mettre en œuvre pour limiter toute

1. Jaboratoire de Zoologie, Domaine Universitaire, B. P. 53, 38031 Grenoble.

L'auteur tínt à remercier $M$. le Professeur $P$. Bourrelly qui a bien voulu l'accueillir en son laboratoire du Muséum Nationale d'Histoire Naturelle afin de l'épauler pour la détermination des algues du lac. 
eutrophisation excessive des deux plans d'eau. Une étude suivie des paramètres physiques et chimiques des eaux (Gay 1976) a mis en évidence le caractère nettement eutrophe du lac de Petichet. Nous nous intéresserons ici au phytoplancton et au zooplancton dont la connaissance de l'abondance relative des espèces dominantes et de leur succession dans le temps et dans l'espace permet d'affiner les résultats des analyses physico-chimiques et de définir les caractéristiques biologiaues qui font l'originalité de ce lac.

\section{1. - LE MILIEU}

Le lac de Petichet, lac glaciaire formé par accumulation des eaux derrière une moraine frontale, est situé sur le plateau de la Mateysine (Isère) à une altitude de $923 \mathrm{~m}$. Il est soumis à un climat de montagne, caractérisé par des hivers froids et longs et une forte amplitude thermique annuelle. Sa superficie est de 87 hectares, son volume de $8700000 \mathrm{~m}^{3}$ et sa profondeur maximale atteint $19,27 \mathrm{~m}$. L'essentiel des apports provient d'un ruisseau de $3,500 \mathrm{~km}$ de long qui traverse un bassin versant à substrat sédimentaire. Le lac de Petichet est utilisé par l'Electricité de France comme lac réservoir et subit des variations dc niveau pouvant atteindre près de $3 \mathrm{~m}$.

Le lac de Petichet est un lac dimictique de second ordre ayant une stratification estivale très nette entre juin et septembre. La transparence moyenne de ses eaux est de $-5,20 \mathrm{~m}$ et sa couleur varie entre le vert et le vert-jaune (catégorie X-XI d'après la gamme de Forel et de Ule). Ses eaux sont moyennement bicarbonatées $(110 \mathrm{mg} / \mathrm{l})$, les teneurs en calcium étant également moyennes $(40 \mathrm{mg} / \mathrm{l})$ et le $\mathrm{pH}$ légèrement basique (valeur moyenne de 8 unités). Durant la stratification estivale, la distribution de l'oxygène est nettement clinograde. La disparition de ce gaz, près du fond, est due à un déficit relatif dans l'hypolimnion de $0,102 \mathrm{mg} / \mathrm{l} /$ jour. Le fer de la vase est alors réduit à l'état d'ions ferreux qui passent en solution dans l'hypolimnion et forment des bicarbonates ferreux solubles au contact d'eau riche en gaz carbonique. Il y a libération par la vase d'ions $\mathrm{PO}_{4}{ }^{:-}$et $\mathrm{NH}_{4}^{+}$et formation d'hydrogène sulfuré qui s'accumule dans le fond. En surface, une active photosynthèse libère de l'oxygène et provoque la diminution des carbonates, nitrates et phosphates. Le $\mathrm{pH}$ est alors nettement basique $(8,50)$ et s'acidifie en profondeur $(7,10)$. Ces phénomènes sont caractéristiques d'un lac eutrophe. Cela est dû en grande partie à l'apport en ions eutrophisants de l'affluent dont les eaux sont polluées par une laiterie-porcherie et de nombreuses fermes, mais également aux variations de niveau du lac qui provoquent, lors de l'inondation des vases exondées depuis plusieurs mois et des débris organiques qui y reposent, la libération de matières organiques et de leurs produits de décomposition. 


\section{2. - METHODE D'ETUDE}

Le plancton servant à l'identification des espèces a été prélevé tous les quinze jours à l'aide d'un filet à mailles de 50 micromètres. Les prélèvements quantitatifs ont été effectués à l'aide d'une bouteille de Friedinger d'une contenance de deux litres pourvue d'un filtre de Zürcher (soie à bluter de 50 micromètres). Ils ont été faits au centre $\mathrm{du}$ lac, aux mêmes profondeurs $(-20 \mathrm{~cm},-2 \mathrm{~m},-5 \mathrm{~m},-10 \mathrm{~m}$ et à $50 \mathrm{~cm} \mathrm{du}$ fond) que les prélèvements d'eau ayant servi aux analyses physico-chimiques. Le plancton fixé a été étudié par comptage dans une cellule de volume connu.

\section{3. - EVOLUTION SAISONNIERE, REPARTITION VERTICALE ET ECOLOGIE DES ESPECES DOMINANTES DU PHYTOPLANCTON}

Une quarantaine d'espèces d'algues ont été recensées dans le lac de Petichet. Nous ne passerons ici en revue que les espèces dominantes et celles ayant une valeur écologique certaine.

\subsection{Bacillariophycées}

Les Diatomées forment l'essentiel du phytoplancton du lac. Elles représentent la presque totalité des algues durant les mois de févriermars et de septembre à décembre (fig. 1-2). Seuls les mois d'avril et de mai, lors de la poussée de Pseudosphaerocystis sp., et le mois de juillet lors de la "fleur d'eau » à Dinobryon divergens, voient leur densité représenter moins de $50 \%$ du total. Trois poussées ont été observées durant l'année 1974. Une première en mai, la plus faible, est due à Melosira italica, une seconde, plus importante, en août, à Fragilaria crotonensis, enfin, une troisième, la plus considérable, en décembre, est le fait d'Asterionella formosa (fig. 6). Parmi les Diatomées trouvées dans le lac, (Asterionella formosa Hass., Cymbella sp., Diatoma elongatum Agardh., Fragilaria crotonensis Kitton, F. virescens Ralfs, Gyrosigma attenuatum Kütz., Melosira italica Kütz., Meridion circulare Agardh., Navicula sp., Nitzschia sp., Pinnularia sp., Synedra acus Kütz., S. ulna (Nitzsch.) Ehr., Tabellaria fenestrata (Lyngb.) Kütz., T. flocculosa (Roth) Dütz.), seules quatre espèces se rencontrent en grande quantité et presque toute l'année (fig. 5).

* Melosira italica semble caractériser le lac. Elle n'atteint jamais les densités de Fragilaria crotonensis ou Asterionella formosa au moment de leur maximum d'abondance. Par contre, elle est présente toute l'année en grande quantité. Sa courbe de croissance montre trois pics: un premier en mai avec 28000 cellules/litre, un deuxième, moins 


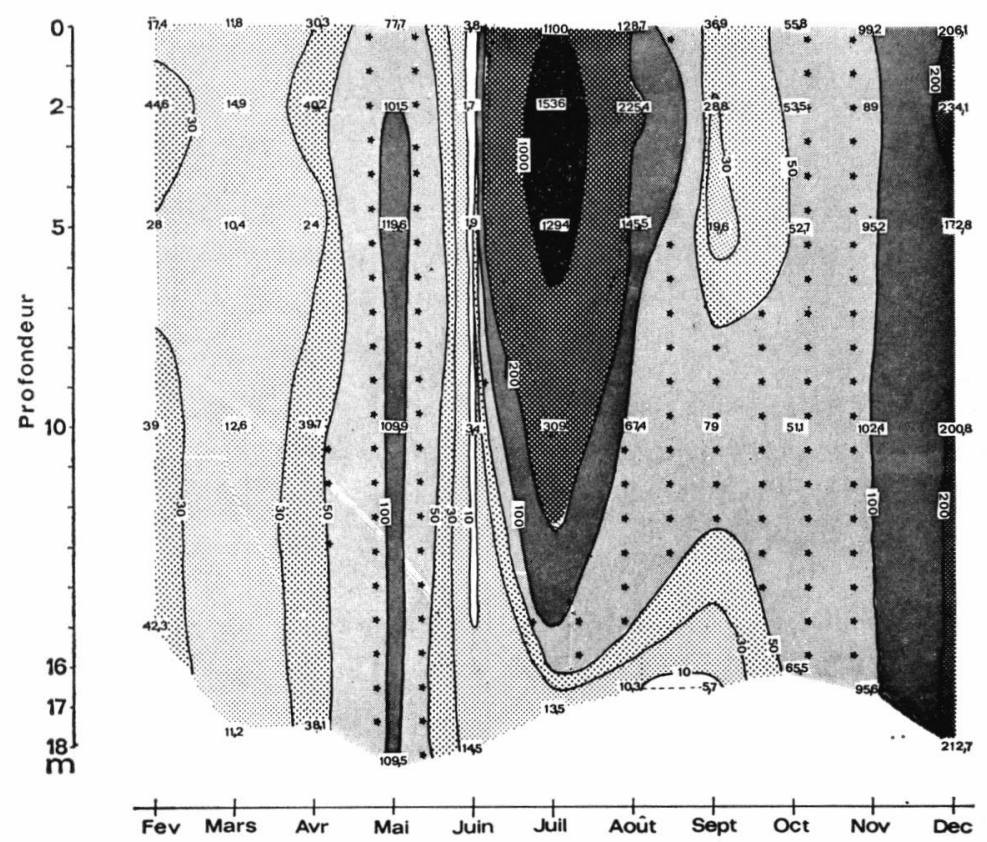

Fig. 1. - Variations au cours de l'année 1974 de l'ensemble des phytoplanctontes. Les chiffres représentent le nombre de cellules par ml d'eau.

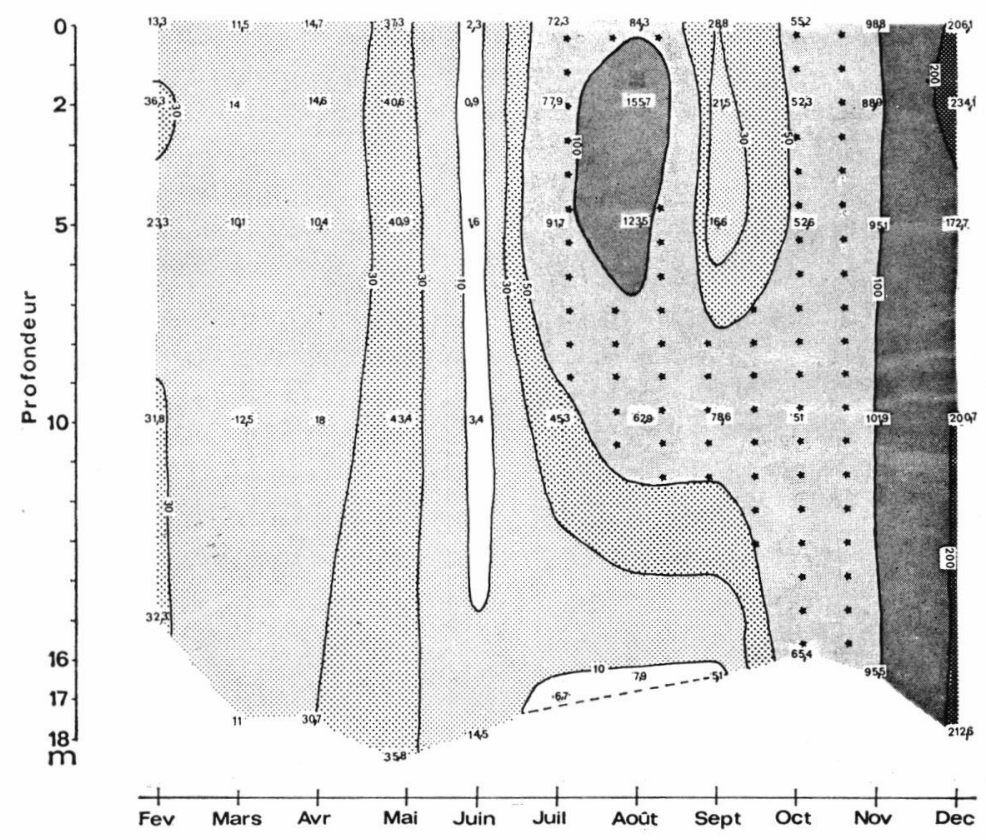

FIg. 2. - Variations au cours de l'année 1974 de l'ensemble des Diatomées. Les chiffres représentent le nombre de cellules par ml d'eau. 


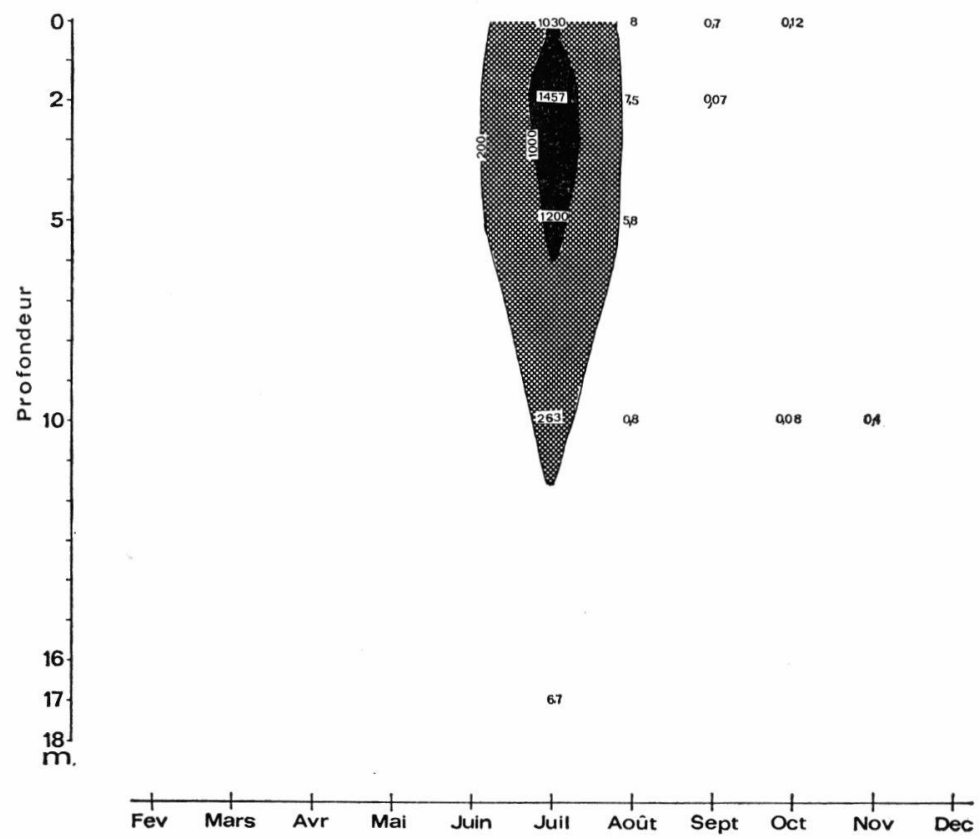

Fig. 3. - Variations au cours de l'année 1974 de Dinobryon divergens. Les chiffres représentent le nombre de cellules par $\mathrm{ml}$ d'eau.

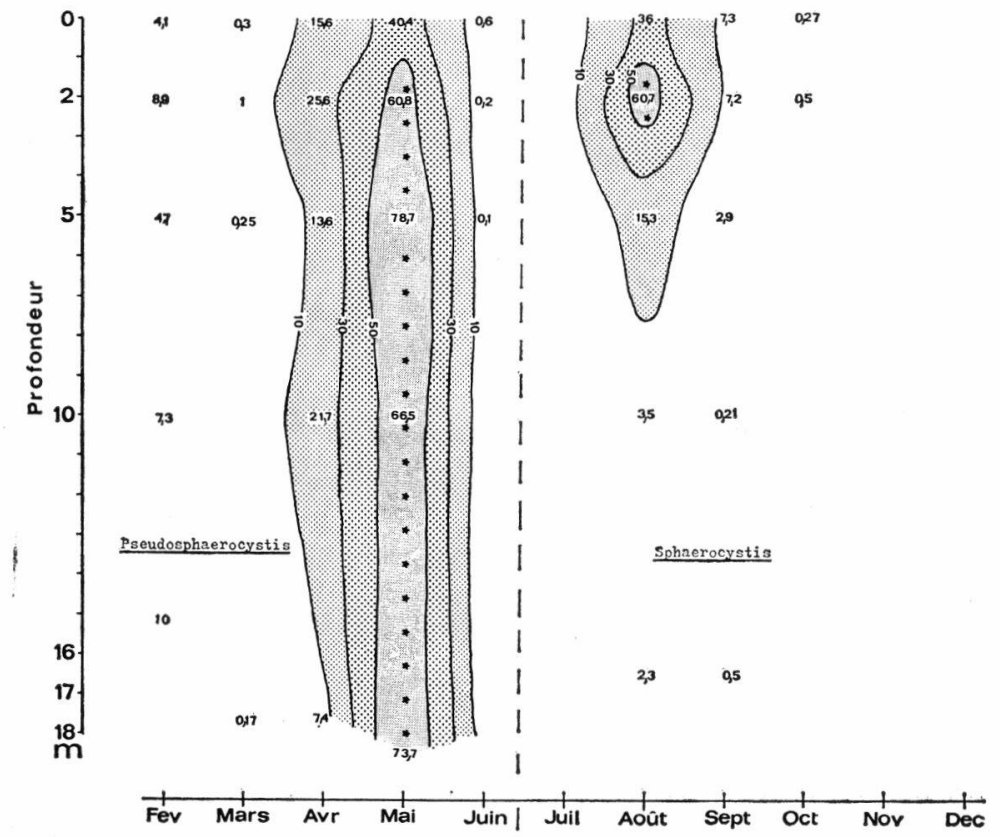

Fig. 4. - Variations au cours de l'année 1974 de Pseudosphaerocystis sp. et de Sphaerocystis schroeteri. Les chiffres représentent le nombre de cellules par ml d'eau. 
important, en juillet ( 15000 cellules/litre à $-2 \mathrm{~m}$ ), enfin un troisième en novembre, le plus considérable, avec 70000 cellules/litre. Le pic du mois de juillet n'est, à mon avis, pas original, car toutes les autres algues présentent ce même phénomène à cette époque de l'année où l'eau est enrichie en ions nutritifs $\left(\mathrm{NO}_{3}{ }^{-}, \mathrm{PO}_{7}{ }^{\circ-}\right)$ et en silicates à la suite de la déplétion du mois de juin (fig. 6). Melosira italica semble donc exiger, pour un développement optimum, une température basse et une faible luminosité. Il est intéressant de remarquer qu'aux mois d'avril et d'octobre, lors des circulations printanières et automnales, cette algue est surtout abondante près du fond (19200 cellules/l à $-17,60 \mathrm{~m}$ contre 9100 à $-10 \mathrm{~m}$ en avril ; 53300 cellules/ $/ \mathrm{à}-16 \mathrm{~m}$ contre 41500 à $-10 \mathrm{~m}$ en octobre). Cela semble en accord avec Lund (1954) qui découvrit que les filaments de Melosira italica ayant sédimenté durant les stagnations d'hiver et d'été pouvaient survivre plusieurs mois sur une vase anaérobie. A la faveur des circulations, ces filaments sont remis en suspension, ce qui donne naissance aux pics des mois de mai et de novembre. Melosira italica est une algue abondante dans les eaux eutrophes.

* Asterionella formosa est une espèce très commune dans de nombreux lacs et pour laquelle les observations sont très nombreuses. Pourtant, son écologie est encore très mal connue. Pour Lefèvre (Vivier, Dussart et al. 1956), elle serait eurytrophe et sténotherme d'eau froide alors que pour M. Feuillade (1969), ce ne serait ni la température ni l'éclairement qui agiraient directement sur la croissance de cette algue, mais certaines substances minérales dissoutes telles que le fer ferreux. Dans le lac de Petichet, elle est présente toute l'année mais n'est abondante qu'en décembre $(167000$ cellules/ 1 à $-2 \mathrm{~m}$ ) quand l'eau est à une température de $3,5^{\circ} \mathrm{C}$. Les teneurs en silice $(2 \mathrm{mg} / \mathrm{l})$ et en phosphates $(0,067 \mathrm{mg} / \mathrm{l})$ sont élevées. Par contre, les teneurs en fer $(0,05 \mathrm{mg} / \mathrm{l})$ ne sont pas particulièrement fortes ainsi que celles en azote nitrique $(0,25 \mathrm{mg} / \mathrm{l})$.

* Fragilaria crotonensis est également présente toute l'année mais elle n'est abondante qu'au mois d'août (151 000 cellules/l à $-2 \mathrm{~m}$ ), dans les cinq premiers mètres du lac, dans une eau dont la température dépasse $20^{\circ} \mathrm{C}$. Bien que souvent considérée comme eurytherme, elle se développe surtout en été dans des eaux chaudes. Dans le lac de Petichet, les teneurs en phosphates sont trop faibles pour être dosables durant les mois de grande abondance de cette algue. D'après Chu (1943), Fragilaria crotonensis et Asterionella formosa seraient des organismes autoauxotrophes, donc capables de produire leurs propres hormones de croissance, d'où une facilité pour la production de "fleurs d'eau". Au mois de septembre, elle se sédimente, mais sa chute est freinée au niveau du métalimnion où les densités de population les plus fortes sont alors observées. 
* Synedra acus est bien moins abondante que les Diatomées précé dentes. Sa densité augmente régulièrement depuis le mois de février pour atteindre un premier maximum en avril ( 600 cellules/1), puis elle se dépose et disparaît totalement en juillet. Elle réapparaît en août, surtout abondante en surface et présente un deuxième maximum en novembre, toutefois plus faible que le premier.

\subsection{Chlorophycées}

Parmi la quinzaine d'espèces trouvées dans le lac, (Coelastrum cambricum var. intermedium Archer., Cosmarium depressum Lund., $C$. punctulatum var. subpunctulatum Börges, Crucigenia rectangularis (Näg.) Gay, Dictyosphaerium pulchellum Wood., Elakatothrix? genevensis Reverd., Geminella interrupta (Turpin) Lagerh., Pediastrum boryanum (Turpin) Menegrini, P. duplex Meyen, Pseudosphaerocystis sp., Scenedesmus spp., Sphaerocystis schroeteri Chodat, Staurastrum avicula Breb., S. cingulum (Smith), seules deux sont abondantes, Pseudosphaerocystis sp. et Sphaerocytis schroeteri. Ces deux genres ont souvent été confondus. Dans le lac de Petichet, Pseudosphaerocystis sp. forme des colonies gélatineuses globuleuses dont les cellules, qui contiennent un plaste étoilé, ont la forme d'une sphère légèrement aplatie à l'un de ses pôles. Sphaerocystis schroeteri forme des colonies globuleuses gélatineuses dont les cellules sphériques, qui contiennent un plaste en forme de coupe, sont distribuées dans la partie centrale de la colonie. Ces deux algues semblent également avoir des exigences différentes (fig. 4 et 6).

Pseudosphaerocystis sp. présente un maximum au mois de mai avec 78700 cellules par litre à $-5 \mathrm{~m}$ ( $66 \%$ du total des algues). Elle est uniformément répartie dans toute la masse d'eau dont la température est de $7,8{ }^{\circ} \mathrm{C}$. Ses exigences en ions nutritifs semblent assez importantes: le développement de cette algue est suivi d'une diminution des phosphates (de $0,010 \mathrm{mg} / 1$ en mars à 0,000 en mai) ainsi que d'une forte baisse du taux de l'azote nitrique (de $0,85 \mathrm{mg} / 1$ en mars à $0,25 \mathrm{mg} / \mathrm{l}$ en mai). Cette algue disparaît totalement en juin pour réapparaître en novembre très faible vers l'automne.

Sphaerocystis schroeteri apparaît brusquement au mois d'août, mois pendant lequel cette algue atteint son maximum de développement (60700 cellules $/ 1$ à $-2 \mathrm{~m} ; 27 \%$ du total des algues). Elle se cantonne dans les premiers mètres du lac où la température de l'eau dépasse $21^{\circ} \mathrm{C}$. Les phosphates ne sont pas dosables et les teneurs en azote nitrique peu importantes $(0,30 \mathrm{mg} / \mathrm{l})$. Elle décroît ensuite très vite à partir de septembre et disparaît totalement en novembre. Il semblerait donc que Pseudosphaerocystis sp. soit une algue d'eau froide ayant des besoins élevés en ions nutritifs, d'où son pic printanier, alors que Sphaerocystis schroeteri serait plutôt une algue d'eau 

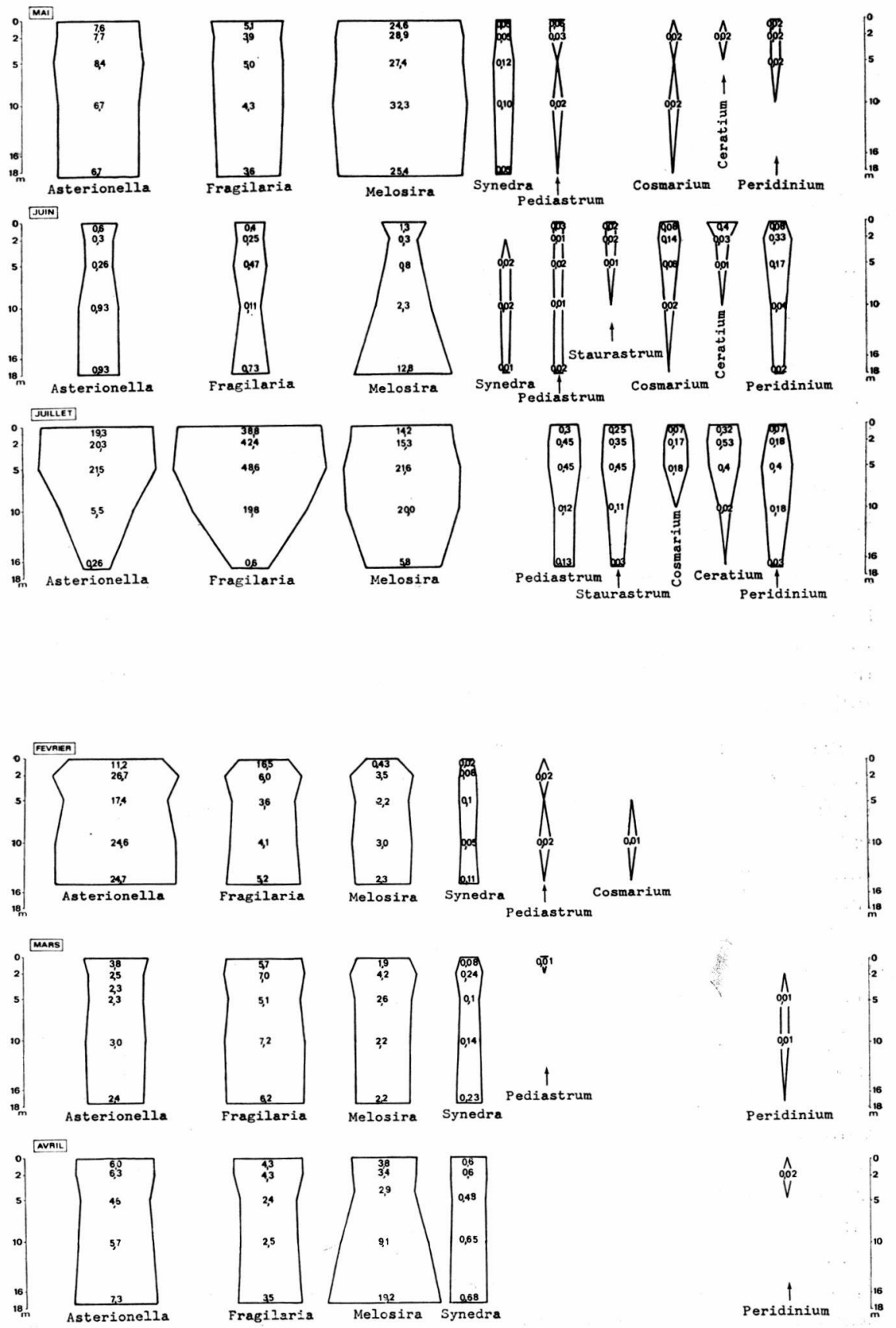
NOVEMBAE
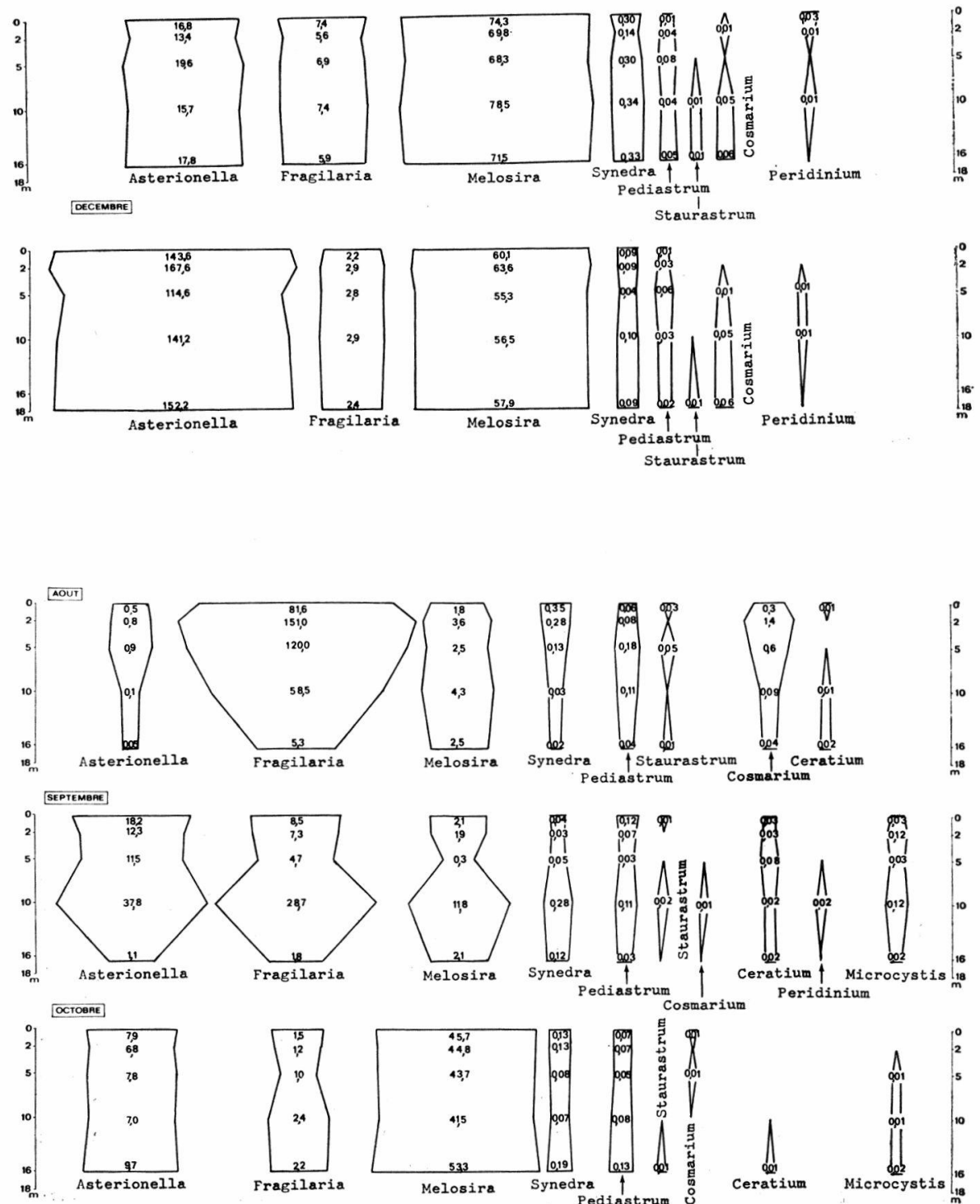
Pediastrum Cosmarium
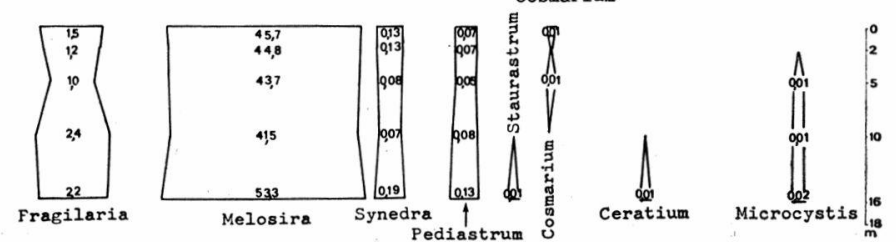

Fig. 5. - Répartition verticale des phytoplanctontes au cours de l'année 1974. Représentation graphique par la méthode de Kohlrausch. Les chiffres indiquent le nombre de cellules (ou de cénobes pour Pediastrum) par $\mathrm{ml}$ d'eau. 
chaude à exigences nutritionnelles peut-être moins importantes. On rencontre cette dernière aussi bien dans les eaux eutrophes que dans les lacs oligotrophes.

Parmi les autres Chlorophycées du lac, Staurastrum cingulum, Cosmarium depressum, Pediastrum duplex et $P$. boryanum sont les seules algues relativement abondantes, bien que ne représentant jamais $1 \%$ de la population phytoplanctonique (fig. 5).

Staurastrum cingulum, algue eulimnoplanctonique, apparaît en surface en juin et montre un maximum de développement en juillet avec 450 cellules/ 1 à $-5 \mathrm{~m}$. Ensuite, elle se dépose et se retrouve occasionnellement vers le fond.

Cosmarium depressum est très rare ou totalement absente du plancton pendant les premiers mois de l'année. Sa densité augmente au printemps pour atteindre son maximum en juillet $(180$ cellules/ 1 à $-5 \mathrm{~m}$ ), date à laquelle l'algue n'est présente qu'en surface.

Pediastrum duplex et $P$. boryanum on été regroupées pour les comptages - les deux espèces étant également représentées - On les trouve durant presque toute l'année, mais elles sont surtout abondantes l'été et, en particulier, en juillet ( 450 cénobes $/ 1$ à $-2 \mathrm{~m}$ ).

Quant à Dictyospharium pulchellum, c'est une algue dont l'apparition est sporadique comme dans les lacs d'Eguzon et de Vezins. (Vivier, Dussart et al. 1956). Elle est souvent abondante avec Pediastrum spp. dans les eaux eutrophes.

\subsection{Chrysophycées}

Dinobryon divergens Imhoff apparaît brutalement en juillet et forme une "fleur d'eau " dans l'épilimnion (1500 cellules $/ \mathrm{ml}$ à $-2 \mathrm{~m}$ ) (fig. 3). Il régresse aussi rapidement et quelques cellules sont encore présentes en novembre. De nombreux auteurs ont étudié les conditions d'apparition de cette algue. Dans tous les cas, cette espèce accroît sa population après le maximum printanier des autres algues, c'est-àdire à une époque où généralement les teneurs en nitrates et surtout en phosphates diminuent. D'après Rodhe (1948), les phosphates seraient même toxiques pour cette espèce à partir d'une concentration de $5 \mathrm{mg} / \mathrm{m}^{3}$. Pourtant, dans les étangs de Sologne, le maximum de densité de Dinobryon divergens coïncide avec des teneurs élevées en phosphates (plus de $0,5 \mathrm{mg} / \mathrm{l}$ de $\mathrm{P}_{2} \mathrm{O}_{5}$; Lefèvre, Jakob et Nisbet 1952). Il en est de même dans le lac de Petichet où cette espèce apparaît après le déclin brutal des autres algues, au moment où les teneurs en phosphates et en nitrates augmentent. En fait, il semble que le principal facteur déterminant la montée des effectifs de $D$. divergens soit la diminution des effectifs des autres espèces. Mais il s'agit là d'un phénomène de compétition interspécifique qui reste à démontrer. 

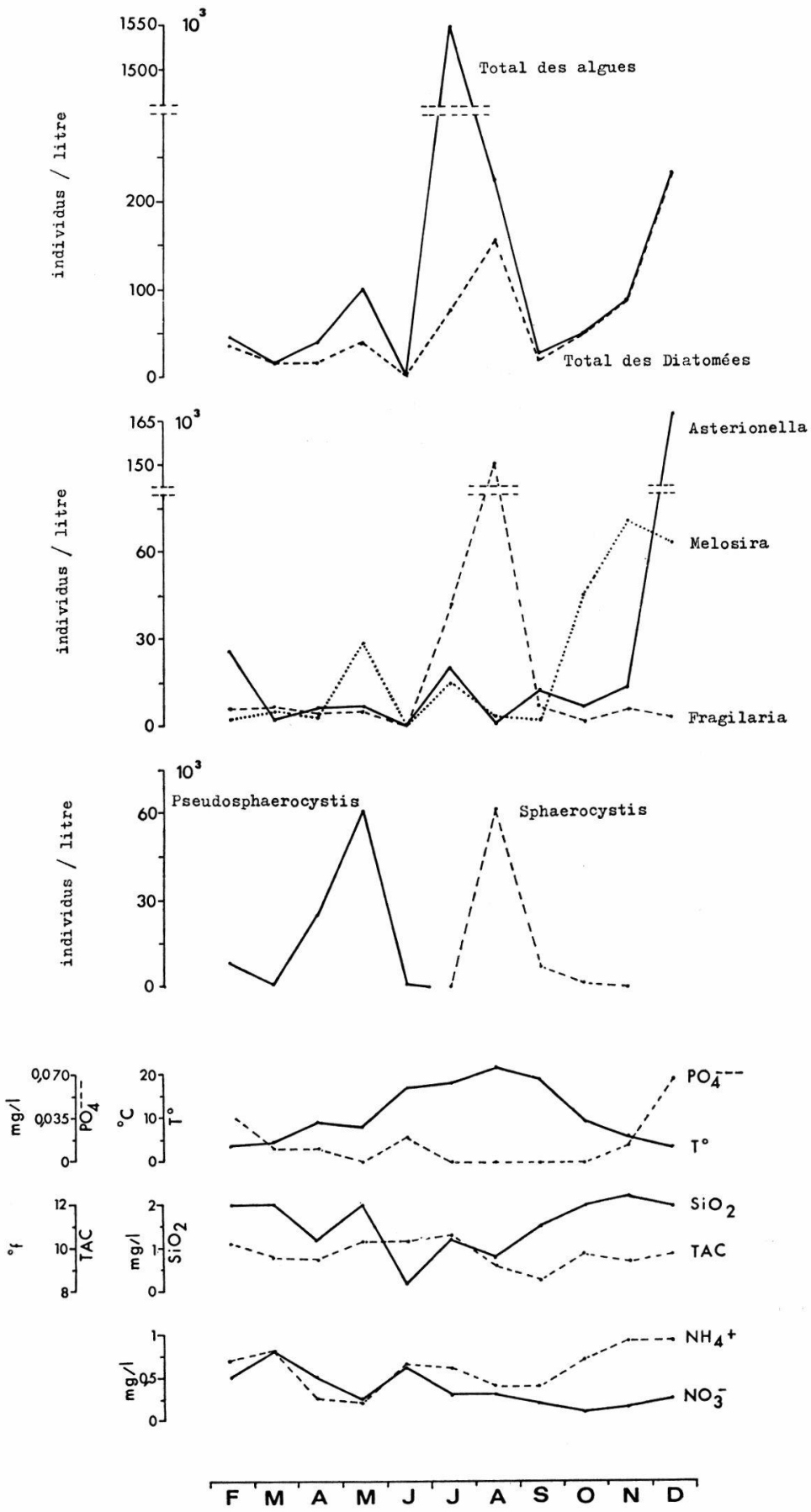

Fig. 6. - Variations des phytoplanctontes et de quelques facteurs physico-chimiques au cours de l'année 1974, à 2 mètres de profondeur. 
$\mathrm{Au}$ mois d'août, après la disparition presque complète de $D$. divergens, D. sociale v. stipitatum (Stein) Lemm. apparaît avec des effectifs assez limités, jusqu'à la fin de l'année. Cette succession d'espèces a déjà été remarquée par Kozarov (in Stankovic 1960) dans le lac Ohrid.

\subsection{Dinophycées}

Ceratium hirundinella (O. F. Müller) Schrank apparaît en surface au mois de mai et devient abondant en juillet et surtout en août (1 400 cellules/l à $-2 \mathrm{~m}$ ). Ensuite, l'algue se dépose et on l'observe pour la dernière fois près du fond en octobre. Cette algue est un euplanctonte pouvant vivre dans toutes sortes de types d'eau, donc donnant très peu de renseignements quant au trophisme du lac. Cela est également vrai de Peridinium volzii Lemm., et $P$. cinctum Ehrbg., les deux Péridiniens que l'on trouve dans le lac de Petichet. Ils ont été regroupés pour les comptages. Leur pic d'abondance se situe entre juin et juillet avec quelques centaines de cellules par litre d'eau (fig. 5).

\subsection{Cyanophycées}

Les algues bleues, souvent abondantes dans les lacs eutrophes, furent assez peu représentées durant l'année 1974. Des exemplaires de Chroococcus minutus (Kg.) Naeg. et C. limneticus Lemm. ont été décelés dans les échantillons durant le mois de juillet, tandis que quelques filaments d'Oscillatoria? cortiana Menegh. se sont développés à l'automne. Enfin, Microcystis aeruginosa Kütz. et $M$. flos-aquae (Wittz.) Kirchn. montrèrent une légère poussée en septembre et octobre. Ces deux algues furent décomptées ensemble (fig. 5). Par contre, un prélèvement effectué en juin 1975 a permis de déceler la formation d'une fleur d'eau à $O$. ? cortiana.

\subsection{Bactéries}

L'examen, à l'aide de microphotographies, de la structure de la paroi des bactéries trouvées en pleine eau, montre que l'on a affaire à des bactéries gram négatif appartenant vraisemblablement à l'ordre des Chlorobactéries. Si cette détermination est correcte, la description correspond à celle donnée par G. Huber-Pestalozzi (1938) pour Schmidlea luteola Lauterborn 1913 (synonyme de Aphanothece luteola Schmidle) : cellules de 1,5 à $2,5 \mu \mathrm{m}$ de longueur sur 1 à $1,5 \mu \mathrm{m}$ de largeur ; colonies de 30 à $300 \mu \mathrm{m}$ de diamètre, en forme de gouttelettes, arrondies ou formées de groupes qui se chevauchent. Un espace clair, ayant l'aspect d'une vacuole, occupe souvent l'intérieur des colonies. C'est une forme similaire à celle de Lamprocystis (bactérie sulfureuse rouge) et de Microscystis aeruginosa (algue bleue). Cette 
bactérie, dont les premières colonies sont apparues mi-juin, est très abondante en juillet, mois durant lequel elle obture les filtres à plancton qui sont alors recouverts d'une gelée jaunâtre. Elle atteint son maximum de développement dans les dix premiers mètres du lac. Elle régresse ensuite au mois d'août mais reste présente en pleine eau, bien que moins abondante, jusqu'en octobre.

\section{4. - LES ESPECES DU ZOOPLANCTON ET LEURS EVOLUTIONS SAISONNIERES}

Deux faits caractérisent le zooplancton du lac de Petichet: d'une part sa pauvreté en espèces, d'autre part les densités exceptionnellement élevées du mois de juin. Les premiers Rotifères et Entomostracés ovigères sont remarqués dès le début du mois d'avril et leur nombre augmente régulièrement, parallèlement à la poussée algale printanière. En juin, on totalise par litre d'eau 2156 Rotifères, 1436 Protozoaires, 102 Copépodes et Nauplii et 62 Cladocères. Ces fortes densités provoquent, par prédation, la presque totale disparition des algues dans le lac et, par la suite, une chute brutale des effectifs de zooplanctontes en juillet.

Le seul Protozoaire abondant du lac est Epistylis rotans Svec., espèce véritablement planctonique. Elle apparaît à la fin du mois de mai et présente un premier maximum de densité en juin avec 1436 individus par litre (température de l'eau de surface : $16^{\circ} \mathrm{C}$ ). Ensuite, elle décroît durant les mois de juillet (740 individus/1) et forme un deuxième maximum en septembre avec 1906 individus par litre (température de l'eau de surface : $18,8^{\circ} \mathrm{C}$ ). Elle a presque entièrement disparu en octobre (30 individus/1). Il est à noter qu'E. rotans est très rare dans l'eau froide de l'hypolimnion. C'est une espèce oligo - à $\beta$ - mésosaprobe (Sladeček 1973).

En hiver, la densité en Rotifères est faible, ceux-ci totalisant 8 individus par litre en février. Cette densité augmente régulièrement dès le mois de mars (100 individus/1), pour atteindre les fortes valeurs de juin. Pendant les sept premiers mois de l'année, trois espèces caractérisent le lac. Ce sont Polyarthra dolichoptera Idelson, $P$. remata Skorikov, toutes deux également représentées et dominant Keratella cochlearis Gosse jusqu'en avril. Cette dernière espèce, plutôt $\beta$. mésosaprobe, représente $95 \%$ des Rotifères du mois de juin. Elles sont accompagnées de formes plus rares telles que :

Keratella quadrata (Müller), espèce des eaux eutrophes, surtout abondante en mai et juin, en particulier dans les couches d'eau profonde du lac. 
Asplanchna priodonta Gosse, forme assez banale, observée de façon sporadique toute l'année, mais surtout abondante en été.

Filinia terminalis (Plate), espèce d'eau froide, apparue de mars à juillet. Mais alors qu'elle était présente dans tout le volume du lac en mars, avril et mai, elle s'est cantonnée dans les eaux froides de l'hypolimnion en juin et juillet.

Kellicottia longispina Kellicott qui, bien que très rare en 1974, formait l'essentiel des Rotifères en mai 1975.

A partir du mois d'août et jusqu'aux mois d'octobre-novembre, les espèces précédentes se font rares et laissent la place à des espèces nouvellement apparues : Synchaeta sp., dont le mauvais état de conservation n'a pas permis de déterminer l'espèce, forme alors l'essentiel du plancton avec Polyarthra major Burckh., Asplanchna priodonta Gosse, Gastropus stylifer Imhoff, Notholca limnetica Carlin et Trichocerca spp.

Les Copépodes sont uniquement représentés par des Cyclopides, aucun Calanoïde n'ayant été trouvé durant cette étude. Cyclops vicinus vicinus Ulian. est l'espèce dominante. Elle se rencontre toute l'année mais elle est surtout abondante au printemps et forme l'essentiel des Crustacés planctoniques en avril et mai (respectivement 17 et 11 individus/l). Cette espèce est, d'après Hutchinson (1967), très répandue dans les lacs eutrophes. En France, elle a été observée dans les lacs d'Auvergne (Olivier 1950) et Dussart l'a trouvé dans les étangs de Sologne et dans le lac d'Eguzon (Vivier, Dussart et al., 1956). Signalons la présence sporadique de Cyclops strenuus Fisch., Cyclops tatricus tatricus Kozm. et Eucyclops serrulatus Fisch.

Les Cladocères sont également représentés par un petit nombre d'espèces. Daphnia longispina, assez rare en hiver, présente un premier maximum de population en juin (62 individus/1) et un second, plus faible, en octobre ( 30 individus/1). Les variétés hyalina f. typica Leydig et longispina ont été décelées, la première étant particulièrement abondante au printemps, la seconde en automne. Parmi les rares autres Cladocères trouvés, citons Bosmina longirostris v. cornuta Jurine, B. longirostris v. originalis (O. F. Müller) et les espèces benthiques Alona affinis (Leydig), A. rectangula Sars et Chydorus sphaericus (O. F. Müller).

\section{5. - CONCLUSION}

Il est souvent difficile de préciser le régime trophique d'un lac à partir des espèces rencontrées dans son plancton. De nombreux auteurs ont tenté de caractériser un écosystème d'eau douce par des 
moyens biologiques : dans ce but, le coefficient de Nygaard (1947) et l'indice de Stockner (1971) sont les plus utilisés. Mais ils sont peu valables car imprécis et inapplicables dans certaines régions. De plus, on note que les populations planctoniques peuvent évoluer d'une année à l'autre, ces changements étant imputables à des variations climatiques. C'est le cas pour Oscillatoria? cortiana qui, très peu représentée en 1974, forme une «fleur d'eau » en juin 1975. De même pour le Rotifère Kellicottia longispina qui, sporadique en 1974, forme l'essentiel du zooplancton au cours du printemps 1975. Enfin, chaque pièce d'eau a ses caractéristiques propres, ce qui rend hasardeuse toute comparaison. Des études portant sur plusieurs années seraient nécessaires pour avoir une image précise des variations annuelles et déterminer si le lac a atteint son équilibre biologique ou s'il montre, au contraire, une évolution vers une eutrophie plus accentuée.

L'eau du lac de Petichet, moyennement pourvue en «ions nutritifs » et suffisamment riche en matières oxydables, permet le développement d'un abondant phytoplancton. Bien qu'un phénomène de "fleur d'eau " à Dinobryon divergens semble se renouveler chaque année, vers le mois de juillet, et qu'une autre "fleur d'eau " à Oscillatoria? cortiana ait été observée durant le mois de juin 1975, il n'en demeure pas moins vrai que le lac de Petichet est plutôt un lac à «Diatomées eutrophes " avec Melosira italica au printemps et à l'automne, Fragilaria crotonensis en été, et Asterionella formosa en hiver. Ces espèces dominent, en certaines saisons, dans de nombreux lacs très productifs de toutes les régions tempérées du monde. Asterionella est la diatomée la plus eutrophe en Grande-Bretagne, tandis que Melosira joue ce rôle en Finlande (Hutchinson 1967). D'autre part, d'après Chu (1943), $A$. formosa et $F$. crotonensis seraient très abondantes lorsque la productivité de l'eau est augmentée par l'action humaine dans le bassin versant.

Le zooplancton, quant à lui, est caractérisé par un petit nombre d'espèces plutôt représentatives d'un étang productif et qui, à certaines périodes de l'année, présentent des densités très fortes.

L'ensemble de ces résultats confirme et précise ceux obtenus à partir des analyses physico-chimiques de l'eau (Gay 1976) et de l'étude des autres représentants de la faune du lac, tels les Diptères Chironomidés et Chaoboridés (Serra-Tosio et Gay 1978).

\section{TRAVAUX CITÉS}

Clit (S. P.). 1943. - The influence of the mineral composition of the medium on the growth of planktonic algae. II. The influence of the concentration of inorganic nitrogen and phosphate phosphorus. J. Ecol., 31 : 109-148.

Feuillade (M.). 1969. - Les varations verticales et saisonnières du phytoplancton du lac de Vézins en 1963. Rech. Hydrobiol. cont., I : 23-50. 
GAY (C.). 1976. - Contribution à l'étude écologique et à l'aménagement d'un lac de montagne : le lac de Petichet (Isère). Thèse de $3^{\mathrm{c}}$ cycle, Grenoble, $124 \mathrm{p}$.

Huber-Pestalozzi (G.). 1938. - Das Phytoplankton Des Süsswassers. Die Binnengewässer, 16 (I) : $342 \mathrm{p}$.

Hutchinson (G. E.). 1967. - A treatise on Limnology, Volume II. Introduction to lake biology and the limnoplankton. John Wiley, New York: $1115 \mathrm{p}$.

LefeVRe (M.), JaKOB (H.) et NiSBet (M.). 1952. - Auto et hétéroantagonisme chez les Algues d'eau douce. Ann. Stat. cent. Hydrobiol. appl., 4 : 5-198.

Lund (J. W. G.). 1954. - The seasonal cycle of the plankton diatom, Melosira italica (Ehr.) Kütz, subsp. subarctica O. Müller. J. Ecol., 42 : 151-179.

Nygaard (G.). 1947. - Hydrobiological studies on some Danish ponds and lakes. Det. Kong. Dansk. Vidensk. Selsk. Bivl. Bkr., 7 (I) : 293 p.

Olivier (L.). 1950. - Contribution à la connaissance de la faune aquatique du Puy-de-Dôme. Faune lacustre. Bull. Soc. Hist. Nat. Auvergne, 48: 111 p.

RoDHE (W.). 1948. - Environmental requirements of freshwater plankton algae. Experimental studies in the ecology of phytoplankton. Symb. bot. upsal., 10 (1) : 1-149.

Serra-Tosio (B.) et GaY (C.). 1978. - Les Diptères Chironomidés et Chaoboridés du lac de Petichet (Isère). Trav. Lab. Hydrobiol., Grenoble, 60-70: 97-105.

SLADEČEK (V.). 1973. - System of Water Quality from the Biological Point of View. Arch. Hydrobiol. Beih., 7 (1-4) : 1-218.

Stankovic (S.). 1960. - The Balkan Lake Ohrid and its living world. Monographiae biol., $9: 357 \mathrm{p}$.

Stockner (J. G.). 1971. - Preliminary characterization of lakes in the experimental lakes area northwester Ontario, using diatom occurences in sediments. $J$. Fish. Res. B. Canada, 28 (2) : 265-275.

Vivier (P.), Dussart (B.) et al. 1956. - Le lac d'Eguzon. Contribution à l'étude physique, chimique et biologique d'un lac de barrage artificiel. Ann. Stat. cent. Hydrobiol. appl., $6: 1-230$.

Février 1980.

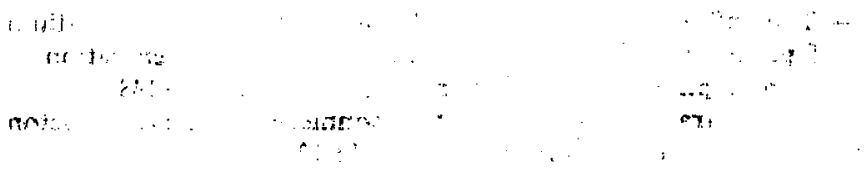

